\title{
The real capabilities of contrast-enhanced ultrasound in the characterization of solid focal liver lesions
}

\author{
Emilio Quaia
}

Received: 1 September 2010 /Revised: 6 October 2010 /Accepted: 7 October 2010/Published online: 24 November 2010

(C) European Society of Radiology 2010

\begin{abstract}
Contrast-enhanced US (CEUS) using intravenous agents is a rapidly evolving field even though the main clinically recognized application is the characterization of focal liver lesions. Several reports have described the improvement provided by CEUS in the characterization of focal liver lesions in comparison to unenhanced US. CEUS with low transmit power insonation allows the real-time assessment of focal liver lesion contrast enhancement and vascularity during the different dynamic phases after injection of an intravenous contrast agent. CEUS allows the accurate characterization of focal liver lesions as benign or malignant based on the lesion contrast enhancement pattern during the arterial phase and lesion vascularity during portal — late phase in comparison to the adjacent liver parenchyma. During the portal-late phase, benign lesions present prevalently a sustained contrast enhancement with hyper or isovascular appearance to the adjacent liver while malignant lesions present prevalently microbubble washout with hypovascular appearance. On the other hand, the histology of focal liver lesions can only be confidently predicted in selected cases by CEUS, as in liver haemangiomas presenting typical nodular peripheral enhancement with subsequent centripetal fill-in and focal nodular hyperplasia with central spoke wheelshaped contrast enhancement.
\end{abstract}

Grey-scale ultrasound (US) is the most widespread imaging procedure for investigating the liver parenchyma due to its relative low cost, availability, safety, and high patient

E. Quaia $(\bowtie)$

Department of Radiology, Cattinara Hospital,

University of Trieste (Italy),

Strada di Fiume 447,

Trieste 34149 , Italy

e-mail: quaia@units.it acceptance. However conventional unenhanced gray-scale US is somewhat limited in characterizing focal liver lesions, and several papers have described the general capabilities of contrast-enhanced US (CEUS) in improving focal liver lesion characterization [1-11].

Recently, European Radiology has published several papers regarding the capabilities of CEUS in focal liver lesion characterization [12-16]. Soussan et al. [16] presented an interesting study evaluating the role of CEUS in the characterization of solid focal liver lesions in comparison to contrast-enhanced MR imaging. In this study authors analysed a series of 47 patients with 50 incidental focal liver lesions-24 focal nodular hyperplasias (FNHs), 11 hepatocellar adenomas, 10 haemangiomas, 1 focal fatty changes, and 4 malignancies including 2 hepatocellular adenomas with malignant foci, 1 hepatocellular carcinoma (HCC), and 1 epithelioid haemangioendotheliomadetected by US and scheduled for contrast-enhanced MR imaging. All patients underwent CEUS after intravenous injection of an US contrast agent consisting of sulphur hexafluoride-filled microbubbles (SonoVue, Bracco, Italy). The MR images and CEUS cine-clips of each lesion were randomly assigned to two blinded readers, with 17 and 22 years of experience in MR imaging and 8 years of experience in CEUS, who independently reviewed all the examinations and were asked to provide the most likely histological diagnosis for each focal liver lesion according to standard diagnostic criteria. A diagnosis with an high level of diagnostic confidence was obtained in $66 \%$ (reader 1 ) and $52 \%$ (reader 2 ) of the lesions for MR imaging compared to $52 \%$ (reader 1 ) and $53 \%$ (reader 2 ) for CEUS. The analysis of CEUS provided an equiviocal diagnosis in 19 lesions according to reader 1 and in 22 lesions according to reader 2 , while the analysis of MR imaging provided a more confident diagnosis respectively in 7/19 lesions according to reader 1 
and in $8 / 22$ lesions according to reader 2 . If the diagnosis of haemangioma was uncertain with CEUS, MR imaging always confirmed the diagnosis. All malignancies were classified with a low confidence score by both readers, except for 1 HCC which was correctly diagnosed by reader 2 after CEUS analysis. If the MR imaging was employed as the first-line imaging technique 14 lesions ( 2 FNHs, 8 hepatocellular adenomas, and the 4 malignant lesions) according to reader 1 , and 24 lesions (9 FNHs, 11 hepatocellular adenomas, and the 4 malignant lesions) according to reader 2 were classified as having an uncertain diagnosis which was improved by CEUS respectively in 5 lesions ( $1 \mathrm{FNHs}$, and the 4 malignant lesions) according to reader 1 , and in 6 lesions ( $5 \mathrm{FNHs}$, and 1 malignant lesion) according to reader 2 . If the diagnosis of FNH was uncertain with either CEUS or MR imaging, the other imaging technique confirmed the diagnosis in approximately half of the cases. The authors concluded that both CEUS and MR imaging have a high diagnostic performance in incidental focal liver lesions and are complementary when the diagnosis is uncertain.

This study [16] also raised the challenging topic of incidental focal liver lesion characterization by CEUS and the potential added diagnostic value provided by CEUS to the other imaging techniques. Focal liver lesions are often identified incidentally during abdominal US performed for other clinical reasons, and frequently these lesions cannot be fully characterized by unenhanced US. Furtehrmore, in a more complex clinical setting, US may identify doubtful focal liver lesions during pre-surgical tumour staging or during post-surgical follow-up, or may detect hepatocellular nodules during surveillance for liver cirrhosis. Unenhanced grey-scale US and colour Doppler US present clear limitations in differentiating malignant from benign liver lesions because of the similar US appearance and vascular architecture of malignant and benign lesions, especially in a background of liver cirrhosis, and are effective in characterizing incidental focal liver lesions as benign or malignant in the normal liver only in $40-50 \%$ of the cases provided that a typical pattern is identified, as in haemangiomas appearing homogeneously hyperechoic or in liver metastases presenting a peripheral hypoechoic halo or also in FNHs presenting a spoke wheel - shaped central vascular pattern at colour Doppler US $[17,18]$. This is mainly due to the well known limitations of Colour Doppler US including colour signal saturation, motion and blooming artefacts, and insensitivity to the slow flow of capillary vessels due to wall filter.

CEUS with microbubble contrast agents and other dedicated US contrast-specific modes were introduced to overcome the limitations of baseline Grey-scale and colour Doppler US [19]. CEUS is not limited by motion and blooming artefacts and allow the real-time assessment of focal liver lesion perfusion during low transmit power insonation. US contrast-specific modes techniques allow a definite improvement in the contrast resolution and suppression of signal from stationary tissues. Perfluorocarbon or sulphur hexafluoride - filled microbubbles covered by a phospholipids shell, including SonoVue and Definity (MRX 115, Bristol-Myers Squibb, New York, NY), present non-linear response with production of harmonic and subharmonic frequencies at low acoustic power insonation (mechanical index from 0.08 up to 0.21 ), allowing the employment of continuous non-destructive imaging and the real-time evaluation of lesion contrast enhancement and vascularity with a better temporal resolution and contrast sensitivity than CT. US contrast agents present a pure intravascular distribution and do not leak in the interstitial space but persist in the sinusoids and portal vessels without the evidence of any equilibrium phase. The enhancement resulting exclusively from the hepatic arterial supply is timed from 10-20 s after the start of the intravenous injection and lasts for $10-15 \mathrm{~s}$. The portal phase then lasts for $2 \mathrm{~min}$ after the start of injection, whereas the subsequent late phase lasts for up to 4-6 min after injection, until microbubble clearance from the liver parenchyma. After blood pool clearance perfulorocarbon-filled agents, such as Sonazoid (NC100100, Nycomed Amersham, Oslo, Norway), were shown to have also hepatosplenic-specific parenchymal affinity at late phase. This phenomenon is probably determined by the adherence of the microbubbles to the hepatic sinusoids or by the selective uptake by the phagocytic cells of the reticuloendothelial system $[20,21]$. Late hepatospecific phase is comparable to liver-specific MR agents, and begins 5 min after i.v. injection lasting from 15 min up to one hour after injection.

CEUS provides a benign or malignant diagnosis based on focal liver lesion enhancement patter at arterial phase and lesion vascularity at portal - late phase in comparison to the adjacent liver parenchyma (Figs. 1 and 2). Malignancies typically show a low echo-signal intensity at late phase $[4,5,8]$, regardless of whether they are hyper- or hypovascular in terms of their arterial supply (Fig. 1). The late phase, with or without hepato-specificity, was showed to be the most important for focal liver lesions characterization since benign lesions present prevalently a sustained enhancement with hyper or isovascular appearance to the adjacent liver while malignant lesions present prevalently microbubble washout with hypovascular appearance [1-15] (Fig. 2).

By excluding patients with a history of cancer and chronic liver disease, Soussan et al. [16] reduced the pretest probability of malignancy in focal liver lesions due to the high prevalence of malignant lesions detected in these patient categories. In this study there is a clear prevalence of benign lesions as it is expected since it includes only incidental focal liver lesions detected in patients without 
Fig. 1 a-d Liver metastases in a 65-year-old man with rectal carcinoma. a Unenhanced grey-scale US. Metastases appear hypoechoic.

b-d CEUS. Contrast specific mode: Cadence Contrast Pulse Sequencing after sulphur hexafluoride-filled microbubble injection with low transmit power insonation. Diffuse contrast enhancement (arrows) is visualized during arterial phase b with lesion hypovascularity during portal c and late phase d. A deeplylocated metastatic lesions is also identificed during the portal and late phase $\mathbf{c}, \mathbf{d}$
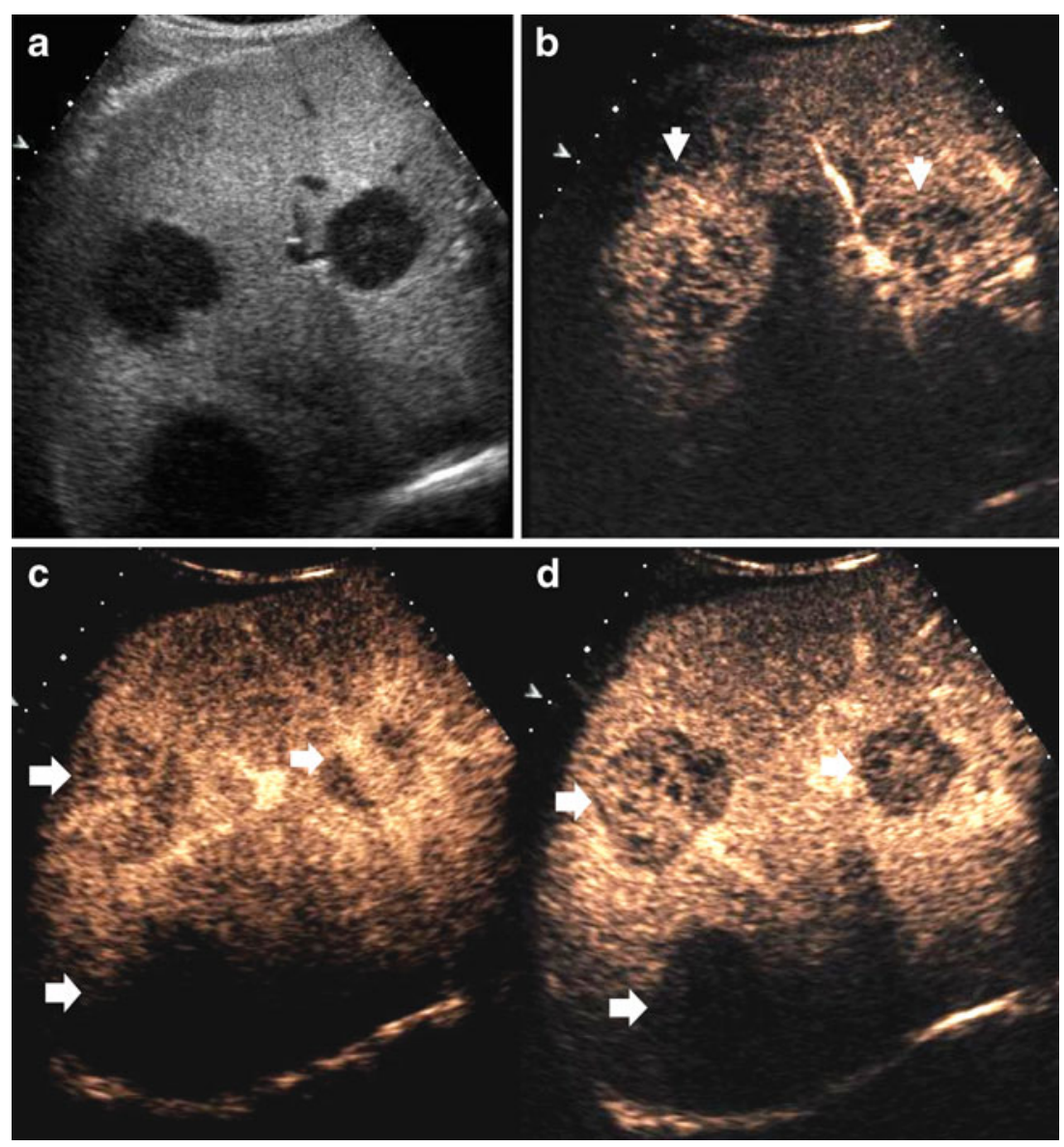

any history of primary cancer or chronic liver disease. This particular clinical setting allowed the authors to attempt to predict a histological diagnosis which is usually not possible in a more diverse patient population including also oncological and cirrhotic patients. Anyway, in routine clinical practice it is often not possible to predict a histological diagnosis by CEUS since many benign lesions appear hypervascular and even after microbubble injection are often indistinguishable; a small hypervascular haemangioma, an FNH, and a hepatocellular adenomas can all appear iso or hypervascular at portal-late phase; the only exception is the hepatocellular adenomas which may appear hypovascular on portal-late phase [22]. Malignant lesions may also not be differentiated since HCCs, cholangiocarcinomas and liver metastases prevalently appear hypervascular during the arterial phase and present washout of contrast agent with hypovascular appearance at portal-late phase, even though HCC may appear isovascular to the adjacent liver parenchyma in about $40 \%$ of cases [6-9]. In such HCCs the isovascular appearance during the portal-late phase may lead to the erroneous impression of a benign lesion, especially if the presence of liver cirrhosis is not known at the time of CEUS examination.
In a recent study including 1034 liver lesions, CEUS yielded a sensitivity of $79.4 \%$ and a specificity of $88.1 \%$ in the differentiation between benign and malignant lesions in oncological or cirrhotic patients [23] which is slight lower if compared to the results reported in a mixed patient population $[2,3,5,8,10]$. In fact the reported sensitivity and specificity of CEUS in the characterization of focal liver lesions in a population including normal, cirrhotic, and oncological patients ranges respectively from $85 \%$ to $90 \%$ and $80 \%$ and $99 \%[3-5,8,10,24]$. The European Federation of Societies for Ultrasound in Medicine and Biology (EFSUMB) guidelines recommends the use of CEUS to diagnose incidental focal liver lesions not characterised on unenhanced US, and in lesions or suspected lesions identified in a background of chronic hepatitis or liver cirrhosis or in patients with a known history of malignancy [25].

In routine clinical practice including normal patients and cirrhotic and oncological patients, CEUS allows to classify liver lesions only as malignant or benign $[2,3,5,8,10]$ without any reference to the final histological diagnosis. In selected cases CEUS may also provide improved lesion histological differential diagnosis provided that a specific enhancement pattern is visualized, as in the case of typical 
Fig. 2 a-d Focal nodular hyperplasia in a 45-year-old woman. a Colour Doppler. Central spoke wheel-shaped pattern (arrows). b-d CEUS. Contrast specific mode: Cadence Contrast Pulse Sequencing after sulphur hexafluoride-filled microbubble injection with low transmit power insonation. Diffuse homogeneous enhancement with hypervascular appearance is evident at arterial phase (arrows) b, persisting at portal $\mathbf{c}$ and late phase $\mathbf{d}$ with lesion (arrows) isovascularity to the adjancent liver parenchyma
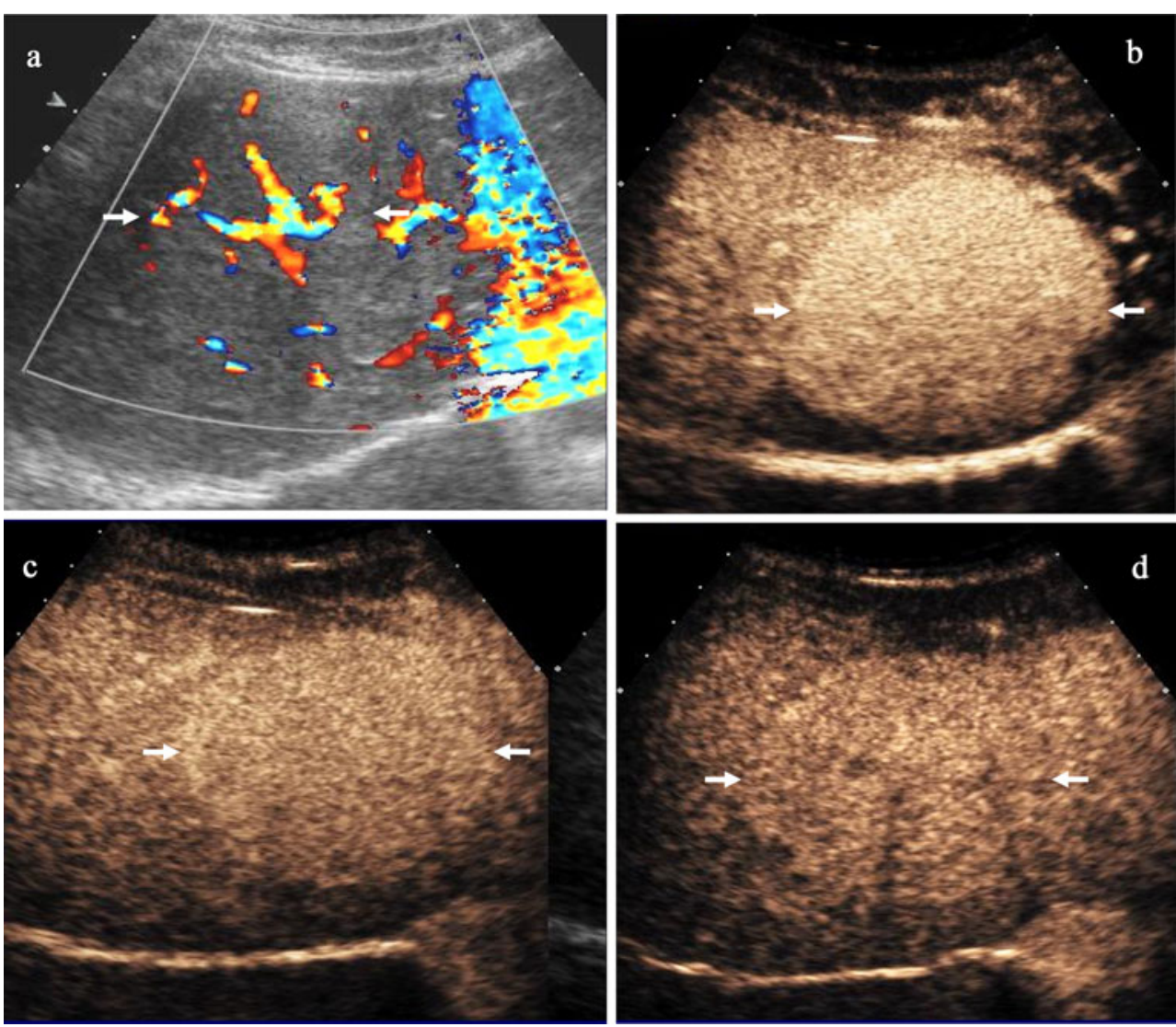

haemangiomas showing peripheral nodular enhancement and FNH showing centrifugal filling with central spoke wheel-shaped contrast enhancement during the arterial phase $[9,10,22]$. Between $5 \%$ and $25 \%$ of focal liver lesions remained indeterminate even after CEUS [2, 3, 5, 8, $10,24]$, since a benign or malignant diagnosis can not be proposed due to the atypical enhancement pattern, and need to be characterized by other diagnostic investigations. Before histological analysis, the two principal investigations necessary to characterize focal liver lesions are CT and MR. It was shown that the combined assessment of hepatocellular nodule vascularity at $\mathrm{CT}$ and CEUS improved diagnostic performance in the diagnosis of malignancy in patients with cirrhosis [26]. Contrast material-enhanced MR with hepatospecific contrast agents, including superparamagnetic iron oxide and gadobenate dimeglumine (Gd-BOPTA) and gadoxetic acid (Gd-EOB-DTPA), allows both dynamic and tissue-specific characterization with improved diagnostic performance in focal liver lesion characterization [27-29].

CEUS is a reliable imaging tool in the characterization of liver lesions with the possibility of prolonged liver ultrasound examination, and is easily performed. The operator has to identify the most suitable acoustic window to evaluate the lesion and simply observe tumour enhancement after the injection of contrast agent without moving the transducer from the initial position. Correct image interpretation is less straightforward and depends on the reader's experience [30]. One limitation of CEUS in comparison to multiphase $\mathrm{CT}$ and MR imaging is the fact that only one liver lesion can be examined at a time as the transducer has to be kept still during the examination, and further contrast injections are necessary to characterize additional liver tumours.

Three-dimensional CEUS creates a spatial and clear depiction of both vascular architecture and hemodynamic features of focal liver lesions [31]. Visual analysis is the simplest method to analyze liver tumour enhancement and vascularity during portal-late phase. However, visual analysis is often penalized in comparison to quantitative analysis by the fact that the observer's eyes tend to focus on a specific portion of the tumour instead of comparing the echogenicity of the whole tumour and adjacent liver in a more global and reproducible manner. The careful retrospective assessment of the recorded video clips may potentially reduce this limitation due to the possibility to assess many times both in detail and as a whole the contrast enhancement dynamics. Nowadays, different real-time automatic computer-aided [32, 33] or parametric quantitative techniques have been developed for distinguishing benign from malignant liver lesions using CEUS and to overcome the limitations of visual analysis. In particular, 
dedicated software can automatically compare the average echo-signal intensity present in a focal liver lesions to the echo-signal intensity in the adjacent liver parenchyma to provide the evidence of persistent microbubble uptake (suggesting a benign lesion) or washout (suggesting a malignant lesion) during the portal-late phase.

In conclusion, CEUS should be considered the logical 'next step' in the characterization of focal liver lesions detected by conventional US. CEUS allows the differentiation of focal liver lesions into benign or malignant, without needing a full histological diagnosis in most of cases.

\section{References}

1. Dill-Macky M, Burns P, Khalili K, Wilson S (2002) Focal hepatic masses: enhancement patterns with SH U 508 A and Pulse Inversion US. Radiology 222:95-102

2. Blomley MJK, Sidhu PL, Cosgrove DO et al (2001) Do different types of liver lesions differ in their uptake of the microbubble contrast agent SH U 508A in the late liver phase? Early experience. Radiology 220:661-667

3. Von Herbay A, Vogt C, Häussinger D (2002) Late-phase pulseinversion sonography using the contrast agent Levovist: differentiation between benign and malignant focal lesions of the liver. Am J Roentgenol 179:1273-1279

4. Brannigan M, Burns PN, Wilson SR (2004) Blood flow patterns in focal liver lesions at microbubble-enhanced US. Radiographics 24:921-935

5. Bryant TH, Blomley MJ, Albrecht T et al (2004) Improved characterization of liver lesions with liver-phase uptake of liver specific microbubbles: prospective multicenter trials. Radiology 232:799-809

6. Nicolau C, Catalá V, Vilana R et al (2004) Evaluation of hepatocellular carcinoma using SonoVue, a second generation ultrasound contrast agent: correlation with cellular differentiation. Eur Radiol 14:1092-1099

7. Giorgio A, Ferraioli G, Tarantino L et al (2004) Contrastenhanced sonographic appearance of hepatocellular carcinoma in patients with cirrhosis: comparison with contrast-enhanced CT appearance. Am J Roentgenol 183:1319-1326

8. Quaia E, Calliada F, Bertolotto M et al (2004) Characterization of focal liver lesions by contrast-specific US modes and a sulfur hexafluoride - filled microbubble contrast agent: diagnostic performance and confidence. Radiology 232:420-430

9. Quaia E, D'Onofrio M, Cabassa P, Vecchiato F, Caffarri S, Pittiani F, Wittkowski K (2007) The diagnostic value of hepatocellular nodule vascularity after sulfur hexafluoridefilled microbubble injection in patients with liver cirrhosis: analysis of diagnostic performance and confidence in malignancy characterization. Am Journal Roentgenology 189:14741483

10. Dai Y, Chen MH, Yin SS, Yan K, Fan ZH, Wu W, Wang YB, Yang W (2007) Focal liver lesions: can SonoVue-enhanced ultrasound be used to differentiate malignant from benign lesions? Invest Radiol 42:596-603

11. Luo W, Numata K, Kondo M et al (2009) Sonazoid-enhanced ultrasonography for evaluation of the enhancement patterns of focal liver tumors in the late phase by intermittent imaging with high mechanical index. J Ultrasound Med 28:439-448
12. Lin MX, Xu HX, Lu MD et al (2009) Diagnostic performance of contrast-enhanced ultrasound for complex cystic focal liver lesions: blinded reader study. Eur Radiol 19:358-369

13. Liu GJ, Xu HX, Xie XY et al (2009) Does the echogenicity of focal liver lesions on baseline gray-scale ultrasound interfere with the diagnostic performance of contrast-enhanced ultrasound. Eur Radiol 19:1214-1222

14. Chen LD, Xu HX, Xie XY et al (2010) Intrahepatic cholangiocarcinoma and hepatocellular carcinoma: differential diagnosis with contrast-enhanced ultrasound. Eur Radiol 20:743-753

15. Bartolotta TV, Taibbi A, Matranga D, Malizia G, Lagalla R, Midiri M (2010) Hepatic focal nodular hyperplasia: contrastenhanced ultrasound findings with emphasis on lesion size, depth, and liver echogenicity. Eur Radiol 20:2248-2256

16. Soussan M, Aubé C, Bahrami S, Boursier J, Charles Valla D, Vilgrain V (2010) Incidental focal solid liver lesions: diagnostic performance of contrast-enhanced ultrasound and MR Imaging. Eur Radiol 20:1715-1725

17. Nino-Murcia M, Ralls PW, Jeffrey RB Jr, Johnson M (1992) Color flow Doppler characterization of focal hepatic lesions. Am J Roentgenol 159:1195-1197

18. Lee MG, Auh YH, Cho KS et al (1996) Color Doppler flow imaging of hepatocellular carcinomas. Comparison with metastatic tumors and hemangiomas by three step grading color hues. Clin Imaging 20:199-203

19. Quaia E (2007) Microbubble ultrasound contrast agents: an update. Eur Radiol 17:1995-2008

20. Quaia E, Blomley MJK, Patel S et al (2002) Initial observations on the effect of irradiation on the liver-specific uptake of Levovist. Eur J Radiol 41:192-199

21. Blomley MJK, Albrecht T, Cosgrove DO et al (1998) Stimulated acoustic emission in liver parenchyma with Levovist. Lancet 351:568-569

22. Kim TK, Jang HJ, Burns PN, Murphy-Lavallee J, Wilson SR (2008) Focal nodular hyperplasia and hepatic adenoma: differentiation with low-mechanical-index contrast-enhanced sonography. Am J Roentgenol 190:58-66

23. Tranquart F, Le Gouge A, Correas JM et al (2008) Role of contrast-enhanced ultrasound in the blinded assessment of focal liver lesions in comparison with MDCT and CEMRI: results from a multicentre trial. Eur J Radiol 6:9-15

24. von Herbay A, Westendorff J, Gregor M (2010) Contrastenhanced ultrasound with SonoVue: differentiation between benign and malignant focal liver lesions in 317 patients. J Clin Ultrasound 38:1-9

25. Claudon M, Cosgrove D, Albrecht T, Bolondi L, Bosio M, Calliada F et al (2008) Guidelines and good clinical practice recommendations for contrast enhanced ultrasound (CEUS) update. Ultraschall in Med 29:28-44

26. Quaia E, Alaimo V, Baratella E, Medeot A, Midiri M, Cova MA (2008) The added diagnostic value of 64-row multidetector CT combined with contrast-enhanced US in the evaluation of hepatocellular nodule vascularity: implications in the diagnosis of malignancy in patients with liver cirrhosis. Eur Radiol 19:651-663

27. Bartolotta TV, Taibbi A, Midiri M, La Grutta L, De Maria M, Lagalla R (2010) Characterisation of focal liver lesions undetermined at grey-scale US: contrast-enhanced US versus 64-row MDCT and MRI with liver-specific contrast agent. Radiol Med 115:714-731

28. Hammerstingl R, Huppertz A, Breuer J et al (2008) Diagnostic efficacy of gadoxetic acid (Primovist)-enhanced MRI and spiral CT for a therapeutic strategy: comparison with intraoperative and histopathologic findings in focal liver lesions. Eur Radiol $18: 457-467$ 
29. Raman SS, Leary C, Bluemke DA et al (2010) Improved characterization of focal liver lesions with liver-specific gadoxetic acid disodium-enhanced magnetic resonance imaging: a multicenter phase 3 clinical trial. J Comput Assist Tomogr 34:163-172

30. Quaia E, Alaimo V, Baratella E, Pizzolato R, Cester G, Medeot A, Cova MA (2010) Effect of observer experience in the differentiation between benign and malignant liver tumors after ultrasound contrast agent injection. J Ultrasound Med 29:25-36

31. Luo W, Numata K, Morimoto $M$ et al (2010) Differentiation of focal liver lesions using three-dimensional ultrasonography: retrospective and prospective studies. World $\mathrm{J}$ Gastroenterol 16:2109-2119

32. Shiraishi J, Sugimoto K, Moriyasu F, Kamiyama N, Doi K (2008) Computer-aided diagnosis for classification of focal liver lesions by use of contrast-enhanced ultrasonography. Med Phys 35:1734-1746

33. Sugimoto K, Shiraishi J, Moriyasu F, Doi K (2009) Computeraided diagnosis of focal liver lesions by use of physicians' subjective classification of echogenic patterns in baseline and contrast-enhanced ultrasonography. Acad Radiol 16:401-411 